\title{
Chapter 5: Australia: the emergence of dual sector universities
}

This chapter describes the use of the term 'dual sector university' in Australia, the characteristics of the universities so described, and speculates why other Australian universities with otherwise similar histories developed as single sector universities. The chapter concludes by considering the potential for the development of new dual sector universities in Australia.

\section{The term 'dual sector university'}

The Australian institutions that identify themselves and recognise each other as dual sector universities are Charles Darwin University, Royal Melbourne Institute of Technology, Swinburne University of Technology, the University of Ballarat and Victoria University in Melbourne. These universities share two characteristics that define them as dual sector universities: they have substantial student load in both vocational education and higher education, and they undertake substantial research and award research doctorates.

\section{'Substantial'}

Dual sector universities have never specified the proportion of load needed in each sector to be considered 'substantial'. The issue can be put rigorously by asking: how high a proportion of total student load must an education sector be before it is no longer considered an exception and it is generally accepted as a normal part of the institution? The same question arises in different contexts: how many women do there have to be in an occupation or worksite before they are no longer considered exceptional but are accepted as part of the norm? Conversely, when do women become so large a part of a workforce that it becomes 'feminised', its wages and working conditions deteriorate and men leave it (Pfeffer \& Davis-Blake, 1987)? A similar dynamic has been observed in the racial segregation of housing in US cities (Grodzins, 1958). Another context is the adoption of an innovation, a new technology, a fad or a new idea. At what point does an innovation pass from the innovators to the early adopters and thence to the early majority (Wilson, 2006)?

Several analytical perspectives have been proposed for these problems: threshold models, bandwagon effects, contagion effects, epidemic theories 
and tipping point. As LaFree (1999: 162) points out, while these concepts vary greatly, all of them assume that under the right circumstances social trends may be nonlinear. The phenomenon has also been studied empirically. The phrase 'tipping point' was coined by Morton Grodzins (1919 - 1964) who studied the racial integration of US neighbourhoods in the early 1960s. He discovered that most white families would remain in a neighbourhood as long as the number of black families remained comparatively small. But at a certain point, when 'one too many' black families arrived, the remaining white families would move out en masse in a process known as white flight. He called that moment the 'tipping point' (Grodzins, 1958). The threshold or tipping point differs for each social trend but 20 per cent is often observed (Wilson, 2006). So for the want of a better alternative one may posit that a university is a dual sector university when at least 20 per cent but no more than 80 per cent of its load is in each sector.

\section{References}

Grodzins, M. (1958). The metropolitan area as a racial problem. Pittsburgh: University of Pittsburgh Press.

LaFree, G. (1999). Declining violent crime rates in the 1990s: predicting crime booms and busts. Annual Review of Sociology, 25, 145-168.

Pfeffer, J. \& Davis-Blake, A. (1987). The effect of the proportion of women on salaries: the case of college administrators. Administrative Science Quarterly, 32, 1-24.

Wilson, C. B. (2006). Adoption of new surgical technology. British Medical Journal, 332, 112-114. 